\title{
ReQuerimientos Ambientales PaRa la Germinación DE Sphaeralcea bonariensis ${ }^{1}$
}

\author{
Environmental Requirements for the Germination of Sphaeralcea bonariensis
}

SOBRERO, M.T. ${ }^{2}$, CHAILA, S. ${ }^{2}$, OCHOA, M.C. ${ }^{2}$ y PECE, M.G. ${ }^{2}$

\begin{abstract}
RESUMEN - S. bonariensis (malva blanca), tolerante a Glifosato, es importante en Argentina en cultivos de siembra directa. En laboratorio se determinó el comportamiento germinativo de la especie en diferentes condiciones de temperatura $(5,10,15,20,25,30,35,40,20 / 10$, $25 / 15$ y $30 / 20{ }^{\circ} \mathrm{C}$ con fotoperiodo de $12 \mathrm{hs}$ de luz); de estrés salino (soluciones de Cloruro de sodio de $0 ; 30 ; 50 ; 70 ; 90 ; 130$ y $150 \mathrm{mM}$ ) e hídrico (soluciones de polyethylene glycol de 0; $-0,2 ;-0,4 ;-0,6 ;-0,8 ;-1,0 \mathrm{MPa})$. El diseño fue completamente aleatorizado con cuatro repeticiones. Se analizó la temperatura con modelos lineales generalizados y prueba DGC para diferencias entre las medias. Para el estrés hídrico y salino se analizó con regresión no lineal. A temperatura constante, la germinación máxima se dio a los 15 y a los $20{ }^{\circ} \mathrm{C}(77$ y $76 \%)$, disminuyó un $49 \%$ a $25{ }^{\circ} \mathrm{C}$, se registraron valores iguales o inferiores al $5 \%$ a 30 y a $35{ }^{\circ} \mathrm{C}$ y fue nula a 5 y a $40{ }^{\circ} \mathrm{C}$. No se observaron diferencias significativas en los porcentajes de germinación en condiciones de alternancia térmica, en todos los casos superó el 50\%. Al incrementar las concentraciones de cloruro de sodio la germinación decreció en forma exponencial. El 66\% de la germinación se registró en el testigo y disminuyó al 10\% a 130 mM, siendo inhibida a $150 \mathrm{mM}$. Cuando el estrés hídrico aumentó de 0 a $-0.6 \mathrm{MPa}$ la germinación decreció del 64\% al 8\% y fue inhibida a $-0.8 \mathrm{MPa}$. La semilla podrá germinar en regiones templadas cálidas, siendo tolerante a la salinidad y medianamente tolerante al estrés hídrico.
\end{abstract}

Palabras-clave: malva blanca, temperatura, potencial osmótico, estrés salino.

\begin{abstract}
S. bonariensis (white mallow), tolerant to glyphosate, is important for the practice of direct sowing in Argentina. The germinating behavior of the species at different temperature conditions (namely 5, 10, 15, 20, 25, 30, 35, 40, 20/10, 25/15, and 30/20 ${ }^{\circ} \mathrm{C}$ in a 12 hour photoperiod of light); saline stress (0,30,50,70,90,130, and $150 \mathrm{mM}$ of sodium chloride solutions); and water stress $(0,-0.2,-0.4,-0.6,-0.8,-1.0 \mathrm{MPa}$ of polyethylene glycol solutions) were determined in the laboratory. The experiment was entirely randomized with four replicates. Temperature was analyzed using generalized digital models, and the DGC test was used to find mean differences. Both water and saline stresses were analyzed by non-lineal regression. At steady temperature, maximum germination occurred at 15 and $20{ }^{\circ} \mathrm{C}$ (77 and $76 \%$ respectively), at $25{ }^{\circ} \mathrm{C}$, it lowered to $49 \%$, at 30 and $35^{\circ} \mathrm{C}$, values equal or lower to 5-30\% were recorded, and germination did not occur at 5 and $40{ }^{\circ} \mathrm{C}$. Significant differences among germination rates were not noted under thermal alternation; all of them were above 50\%. Germination rates decreased exponentially with increased sodium chloride concentrations. Control provided 66\% of germination, which lowered to $10 \%$ at $130 \mathrm{mM}$, and was inhibited at $150 \mathrm{mM}$. When water stress was increased from 0 to -0.6 MPa, germination rate decreased from $64 \%$ to $8 \%$, and was inhibited at $-0.8 \mathrm{MPa}$. Seeds will be able to germinate in warm-temperate regions, being tolerant to salinity and moderately tolerant to water stress.
\end{abstract}

Keywords: white mallow, temperature, osmotic potential, salinity stress.

Recebido para publicação em 30.12.2013 e aprovado em 7.4.2014.

Universidad Nacional de Santiago del Estero, Santiago del Estero, Argentina, <catedra_matologia@unse.edu.ar>.

Planta Daninha, Viçosa-MG, v. 32, n. 3, p. 491-496, 2014 


\section{INTRODUCCIÓN}

Sphaeralcea bonariensis (Malvaceae SPHBO), es conocida vulgarmente como malva blanca, malva, malva del zorro. Nativa de América, se la encuentra en Argentina, Bolivia, Brasil, Chile, Paraguay y Uruguay. En Argentina está muy difundida en las provincias del noroeste, noreste y centro del país. Planta perenne, subarbustiva de 1,0 a $1,5 \mathrm{~m}$ de altura, de porte erecto, con tallos ramificados desde la base cubiertos de pelos estrellados grisáceos al igual que las hojas. Vegeta desde la primavera, fructifica hasta el otoño y se propaga por semillas (Marzocca, 1994; Sobrero \& Chaila, 2006; Nisensohn et al., 2007).

S. bonariensis ha adquirido creciente importancia en el noreste de la Argentina dado que, al igual que otras especies presentes en borduras y alambrados, pasó de ser una especie ruderal a estar presente en barbechos, en cultivos de siembra directa y además por ser mencionada como tolerante al herbicida glifosato (Rodriguez \& Rainero, 2004; Sobrero $\&$ Chaila, 2006; Ledda, 2011).

Comprender la biología de la semilla de una maleza es el punto de partida para desarrollar sistemas de manejo integrado (Chauhan \& Johnson, 2009). La germinación de semillas y emergencia de plántulas son eventos claves que determinan el éxito de una maleza en un agroecosistema, un proceso regulado por factores intrínsecos como cubierta seminal impermeable, presencia de inhibidores, existencia de embriones inmaduros y por factores extrinsecos tales como temperatura, luz, humedad, pH y salinidad del suelo (Forcella et al., 2000). La temperatura es uno de los principales factores que controla la germinación, actúa sobre las enzimas que intervienen en este proceso, afectando tanto la tasa como el porcentaje final de germinación (Bewley \& Black, 1994). La habilidad para germinar bajo condiciones de estrés hídrico y salino en el suelo puede, darle ventaja a una maleza que se vale de esa situación. Poco se conoce sobre la biología de $S$. bonariensis, especialmente sobre los aspectos relacionados con su germinación. La disponibilidad de este tipo de información es importante para determinar la verdadera problemática de la maleza y desarrollar programas de control. El objetivo de este trabajo fue determinar la influencia de las temperaturas continuas y alternas y el efecto del estrés salino e hídrico sobre la germinación de $S$. bonariensis.

\section{MATERIALES Y MÉTODOS}

En febrero de 2013, se cosecharon frutos maduros de $S$. bonariensis en la localidad de El Zanjón, Santiago del Estero, Argentina

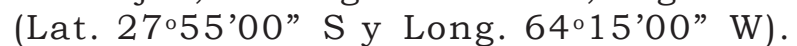
En laboratorio se separaron las semillas de los frutos mediante fricción, las cuales se conservaron en bolsas de papel a temperatura ambiente hasta el inicio de los ensayos.

Para evaluar la germinación, se colocaron semillas previamente escarificadas con lija en placas de Petri, conteniendo dos capas de papel de filtro, humedecidos con agua destilada, soluciones de cloruro de sodio o de polyethylene glycol 6000 (PEG) según correspondiere. La incubación se efectuó en cámara de crecimiento. Se registró la germinación diariamente, durante 14 días. La semilla se consideró germinada cuando el tamaño de la radícula alcanzó 1 1/2 el tamaño de la semilla.

Para evaluar el estrés salino e hídrico se renovó cada dos días la solución de polyethylene glycol o cloruro de sodio, cambiándose los papeles de filtro.

\section{Ensayo de temperaturas continuas y alternas}

Se colocaron veinticinco semillas por tratamiento en cámaras de crecimiento a temperaturas constantes de $5 ; 10 ; 15 ; 20 ; 25$; $30 ; 35$ y $40{ }^{\circ} \mathrm{C} \pm 2{ }^{\circ} \mathrm{C}$ y a temperaturas alternas de $20 / 10 ; 25 / 15$ y $30 / 20{ }^{\circ} \mathrm{C} \pm 2{ }^{\circ} \mathrm{C}$ con un fotoperiodo de $12 \mathrm{hs}$ de luz.

\section{Efecto del estrés salino e hídrico}

Para determinar el efecto de la salinidad sobre la germinación se incubaron veinticinco semillas por tratamiento en soluciones de cloruro de sodio de $0 ; 30 ; 50 ; 70 ; 90 ; 130$ y $150 \mathrm{mM}$.

Para evaluar los efectos del estrés hídrico se prepararon soluciones acuosas con potenciales osmóticos de $0 ;-0.2 ;-0.4 ;-0.6 ;-0.8$, 
-1.0 MPa, disolviendo cantidades apropiadas de PEG 6000 (polyethylene glycol 6000) en agua destilada (Michel, 1983). Los rangos de cloruro de sodio y de potenciales osmóticos reflejan los niveles de salinidad y el estrés hídrico en la zona. La incubación se efectuó en cámara de crecimiento a $20^{\circ} \mathrm{C}$ y con un fotoperiodo de 12 hs de luz.

\section{Diseño experimental y análisis de resultados}

Los ensayos se realizaron con un diseño completamente aleatorizado con cuatro repeticiones por tratamiento. Los datos de temperatura se analizaron con modelos lineales generalizados. Se empleó la distribución binomial para modelar la variable número de semillas germinadas y para el predictor lineal se consideró la temperatura como efecto fijo utilizándose la función de enlace logit (Crawley, 2007). Las diferencias entre las medias de los tratamientos se realizaron mediante la prueba DGC $(\mathrm{p}=0.05)$. Los resultados de los ensayos de estrés salino e hídrico se analizaron mediante regresión no lineal. El procesamiento de los datos se efectuó mediante el paquete estadístico INFOSTAT (Di Rienzo et al., 2013).

\section{RESULTADOS Y DISCUSIÓN}

\section{Efecto de temperaturas continuas y alternas}

Se obtuvo una germinación mayor al 50\% a 15 y a $20{ }^{\circ} \mathrm{C}$ (77 y $\left.76 \%\right)$, esta disminuyó al $49 \%$ cuando fueron incubadas a $25^{\circ} \mathrm{C}$, se registraron valores iguales o inferiores al $5 \%$ a 30 y a $35^{\circ} \mathrm{C}$ y fue nula a 5 y $40{ }^{\circ} \mathrm{C}$, por lo cual estas temperaturas podrian considerarse como detrimentales. En todas las temperaturas alternas ensayadas el porcentaje de germinación superó el 50\% (Figura 1).

La temperatura es considerada un importante factor en la germinación de las semillas de malezas. Ha sido motivo de numerosos estudios en casi todas las especies importantes para los cultivos, no así para $S$. bonariensis.

La respuesta a la temperatura para $S$. bonariensis es similar a las registradas para
Sida spinosa y S. rhombifolia. La especie ensayada germinó en el rango de 20 a $40{ }^{\circ} \mathrm{C}$ mientras que $S$. rhombifolia entre 20 a $35^{\circ} \mathrm{C}$. S. rhombifolia germinó mejor que $S$. spinosa a 20 y $25^{\circ} \mathrm{C}$ pero no germinaron a $40{ }^{\circ} \mathrm{C}$ (Smith et al., 1992). Sin embargo, para Sida spinosa Baskin \& Baskin (1984) registró que las temperaturas alternas $30 / 15,35 / 20$ y $40 / 25{ }^{\circ} \mathrm{C}$ en condiciones de alternancia lumínica, favorecieron la germinación con porcentajes que superaron el 16, el 25 y el 32\% respectivamente. En Hibiscus trionum no se registró germinación a 10 y $45^{\circ} \mathrm{C}$, sin embargo en $S$. bonariensis se registró germinación a $10^{\circ} \mathrm{C}$ y fue nula a $40{ }^{\circ} \mathrm{C}$; en las temperaturas alternas los valores de germinación para $S$. bonariensis superaron las registradas por $H$. trionum que fueron del $60 \%$ para $30 / 20{ }^{\circ} \mathrm{C}$ y del 40 para $20 / 10{ }^{\circ} \mathrm{C}$ (Chachalis et al., 2008). Para Sphaeralcea coccinea se registraron como temperaturas óptimas de germinación 15, 20 y $30 / 15^{\circ} \mathrm{C}$, para Malva moschata 15,20 y $30 / 20{ }^{\circ} \mathrm{C}$ (AOSA, 2007) y para Anoda cristata $30{ }^{\circ} \mathrm{C}$ (Solano et al., 1976). Para Abutilon theophrasti la máxima germinación se registró entre 10 y $40{ }^{\circ} \mathrm{C}$ y declinó a $45^{\circ} \mathrm{C}$ (Sadeghloo et al., 2013). Las temperaturas en las que germinó $S$. bonariensis se encuentran dentro del rango de las especies citadas anteriormente.

Las temperaturas óptimas de germinación de $S$. bonariensis son similares a las registradas en la región del estudio a principios del otoño y desde fines del invierno al inicio de la primavera; esto es coincidente con lo observado por Ledda (2011) sobre la emergencia de plántulas en campo. Esto le otorgaría a $S$. bonariensis una ventaja competitiva sobre otras especies de malezas que requieren temperaturas más elevadas para la germinación. Esta flexibilidad en la germinación provee muchas oportunidades para la producción de semillas y proliferación de la maleza.

\section{Efecto del estrés salino e hídrico}

La germinación de $S$. bonariensis decreció en forma exponencial al incrementar las concentraciones de cloruro de sodio de 0 a $150 \mathrm{mM}$, por lo que los valores obtenidos para los parámetros de la función mediante regresión no lineal, se expresan como: $\mathrm{y}=$ 70,79. exp ${ }^{(0,00153 . x)}$ siendo el cuadrado medio 


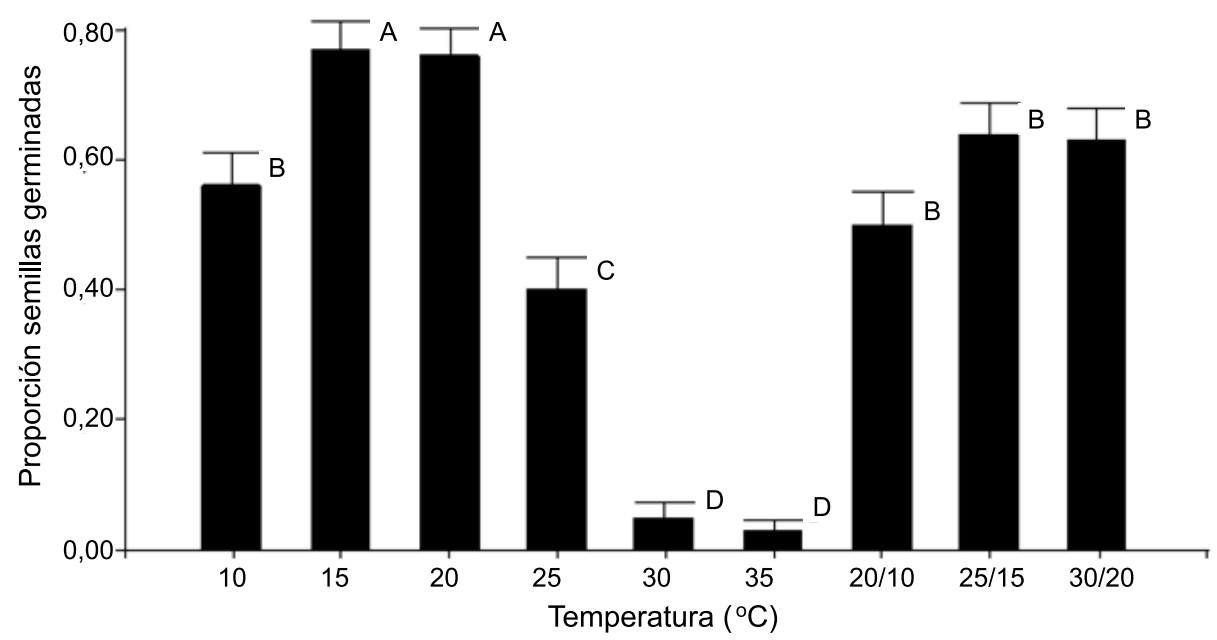

Figura 1 - Efecto de las temperaturas constantes y alternas sobre la proporción de semillas germinadas de S. bonariensis.

del error 122,35 y los valores de AIC y BIC de 179,74 y 183,14 , respectivamente. E1 porcentaje fue mayor (66\%) en el testigo y disminuyó al 10\% a 130 mM. La germinación fue completamente inhibida a $150 \mathrm{mM}$ (Figura 2).

Estos resultados sugieren que S. bonariensis, aunque sensible a la salinidad, puede tolerar concentraciones salinas más altas que Bidens pilosa (Reddy \& Singh, 1992) y Eupatorium adenophorum (Lu et al., 2006), malezas que germinaron hasta $1,0 \mathrm{mM}$. La germinación de Sonchus oleraceus fue mayor al $90 \%$ a $40 \mathrm{mM}$ y a $160 \mathrm{mM}$ fue solamente de un $7,5 \%$ (Chauhan et al., 2006). Para Conyza canadensis, Nandula et al., (2006) obtuvo porcentajes de germinación mayores al $20 \%$ a concentraciones menores de $40 \mathrm{mM}$ de cloruro de sodio y menores al 4\% a $160 \mathrm{mM}$. La germinación de Galium tricornutum fue mayor que el $44 \%$ a concentraciones menores de $20 \mathrm{mM}$ y del $2 \%$ a $160 \mathrm{mM}$ (Chauhan et al., 2006). Mimosa pudica e Ipomoea asarifolia respondieron en diferentes grados de intensidad a la variación de la concentración salina. Para Mimosa la germinación estuvo siempre por encima del $90 \%$ en el intervalo de 0 a $150 \mathrm{mM}$, mientras que para Ipomoea la germinación fue superior al $80 \%$ en el intervalo de 0 a $25 \mathrm{~mm}$ (Souza Filho et al., 2001). En Abutilon theophrastilos porcentajes de germinación fueron de1 $74-89 \%$ en concentraciones de $45 \mathrm{mM}$ de cloruro de sodio, la germinación disminuyó con el incremento de la concentración salina siendo nula a 225 mM (Sadeghloo et al., 2013).

Los resultados obtenidos sugieren que $S$. bonariensis es tolerante a la salinidad en el proceso de germinación llegando a germinar hasta $130 \mathrm{mM}$.

Similarmente a los resultados de los experimentos de estrés salino, la germinación decreció del $64 \%$ al 8\% cuando el estrés hídrico aumentó de 0 a $-0.6 \mathrm{MPa}$. La germinación fue completamente inhibida a $-0.8 \mathrm{MPa}$ y superiores (Figura 3). La función que relaciona el porcentaje de germinación con el

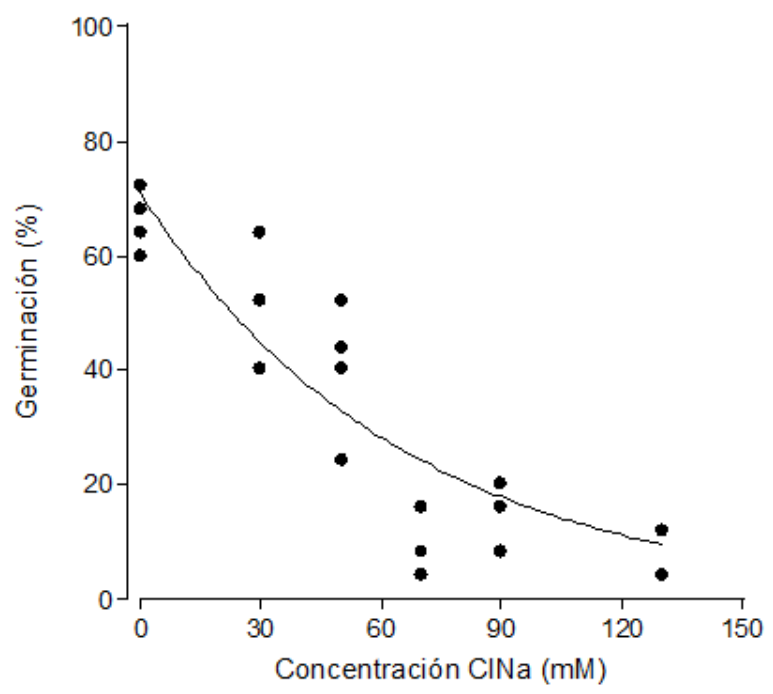

Figura 2 - Efecto de concentraciones de cloruro de sodio sobre la germinación de $S$. bonariensis. 
potencial osmótico es lineal con pendiente negativa y sus valores se expresan con la siguiente ecuación:

$\%$ germinación $=61,40-9,40 *$ tratamiento

Los parámetros de ajuste correspondientes a la misma son: $\mathrm{R}^{2}$ ajustado $=0,89$; $\mathrm{AIC}$ $=113,98 ; \mathrm{BIC}=116,30$, consiguiendo una distribución de residuos sin patrón alguno.

Los potenciales osmóticos obtenidos para otras especies como $S$. rhombifolia y $S$. spinosa (0 a -0,8 MPa y 0 a -0,6 MPa respectivamente) fueron similares a las de $S$. bonariensis (Smith et al., 1992). En Abutilon theophrastila germinación fue completamente inhibida a 0,6 MPa (Sadeghloo et al., 2013). En Hibiscus trionum la germinación fue del $22 \%$ a potenciales osmóticos superiores a $-0.8 \mathrm{MPa}$ $\mathrm{y}$ fue totalmente inhibida a potenciales osmóticos menores a $-1.3 \mathrm{MPa}$, lo cual indicaría que $H$. trionium es tolerante a bajos potenciales hídricos (Chachalis et al., 2008), estos valores son superiores a los registrados para $S$. bonariensis. Sin embargo, $S$. bonariensis mostró mayor sensibilidad al estrés hídrico que Carduus nutans, Ipomoea lacunosa, Mimosa invisa, que germinaron hasta -1.0 MPa (Medd \& Lovett, 1978; Oliveira \& Norsworthy, 2006; Chauhan \& Johnson, 2008). Estudios realizados por Zhang et al. (2005) y Kleemann et al. (2007), muestran que malezas como, Artemisia ordosica y

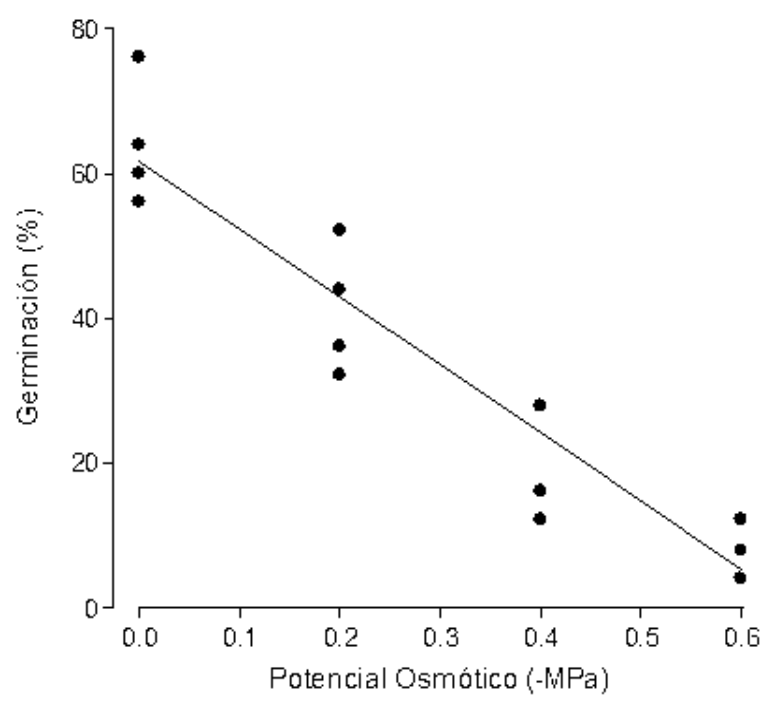

Figura 3 - Influencia del potencial osmótico sobre el porcentaje de germinación de $S$. bonariensis.
Diplotaxis tenuifolia germinaban hasta 1.4 MPa. En contraste, otras malezas como Campsis radicans, y Diodia virginiana demostraron ser altamente sensibles al estrés hídrico y no germinaron a potenciales de $0.2 \mathrm{MPa}$ (Baird \& Dickens, 1991; Chachalis \& Reddy, 2000). La habilidad de S. bonariensis de germinar hasta potenciales de -0.6 MPa indica que la maleza es capaz de establecerse bajo condiciones de suelos de bajo contenido de humedad. La tolerancia al estrés hídrico de una pequeña proporción de las semillas del banco de malezas durante la germinación, puede ser benéfica para su establecimiento en el campo después de que ocurran las lluvias de primavera.

Con este estudio se ha comprobado que $S$. bonariensis está adaptada a un amplio rango de condiciones ambientales, demostrando ser medianamente tolerante al estrés hídrico, tolerante al estrés salino y tener una habilidad manifiesta para germinar entre 10 y $35^{\circ} \mathrm{C}$. Estas características le permiten estar presente en regiones templadas cálidas y establecerse en cultivos primaveroestivales.

\section{AGRADECIMIENTOS}

Al Consejo de Investigaciones Científicas y Tecnológicas de la Universidad Nacional de Santiago del Estero por el apoyo económico para la realización de este trabajo.

\section{LITERATURA CITADA}

BAIRD, J. M.; DICKENS, R. Germination and emergence of Virginia buttonweed (Diodia virginiana). Weed Sci., v. 39, n. 1, p. 37-41, 1991.

BASKIN, J. M.; BASKIN, C. C. Environmental Conditions Required for Germination of Prickly Sida (Sida spinosa) Weed Sci., v. 32, n. 6, p. 786-791, 1984.

BEWLEY, J. D.; BLACK, M. Seeds: physiology of development and germination. 2.ed. New York: Plenum Press, 1994. 445 p.

CHACHALIS, D.; REDDY, K. N. Factors affecting Campsis radicans seed germination and sedling emergence. Weed Sci., v. 48, n. 2, p. 212-216, 2000.

CHACHALIS, D. et al. Factors affecting seed germination and emergence of Venice Mallow (Hibiscus trionum) Weed Sci., v. 56, n. 4, p. 509-515, 2008. 
CHAUHAN, B. S. et al. Factors affecting seed germination of threehorn bedstraw (Galium tricornutum) in Australia. Weed Sci., v. 54, n. 3, p. 471-477, 2006.

CHAUHAN, B. S. et al. Factors affecting seed germination of annual sowthistle (Sonchus oleraceus) in southern Australia. Weed Sci., v. 54, n. 5, p. 854-860, 2006.

CHAUHAN, B. S.; JOHNSON, D. E. Seed germination and seedling emergence of Giant sensitiveplant (Mimosa invisa). Weed Sci., v. 56, n. 2, p. 244-248, 2008.

CHAUHAN, B. S.; JOHNSON, D. E. Germination ecology of spiny (Amaranthus spinosus) and slender amaranth (A. viridis): Troublesome weeds of direct-seeded rice. Weed Sci., v. 57, n. 4, p. 379-385, 2009.

CRAWLEY, M. J. The R book. Chichester: John Wiley \& Sons, 2007. 942 p.

DI RIENZO J. A. et al. InfoStat versión 2013. Grupo InfoStat, FCA, Universidad Nacional de Córdoba, Argentina. URL http://www.infostat.com.ar. 2013.

FORCELLA, F. et al. Modeling seedling emergence. Field Crops Res., v. 67, n. 2, p. 123-139, 2000.

KLEEMANN, S. G. L. et al. Factors affecting seed germination of perennial wall rocket (Diplotaxis tenuifolia) in Southern Australis. Weed Sci., v. 55, n. 5, p. 481-485, 2007.

LEDDA, A. Persistencia de Sphaeralcea bonariensis Cav. Griseb. (Malva blanca): comportamiento germinativo, fenología y respuesta al herbicida glifosato. 2011. Tesis (Grado de Magister) - Facultad de Ciencias Agrarias. Universidad Nacional del Nordeste, Corrientes, Argentina, 2011.

LU, P. et al. Effects of environmental factors on germination and emergence of Crofton weed (Eupatorium adenophorum). Weed Sci., v. 54, n. 3, p. 452-457, 2006.

MARZOCCA, A. Manual de malezas Buenos Aires: Hemisferio Sur, 1994. 564 p.

MEDD, R. W.; LOVETT, J. V. Biological studies of Carduus nutans (L.) spp nutans. 1. Germination and light requirement of seedlings. Weed Res., v. 18, n. 6, p. 363-367, 1978.
MICHEL, B. E. Evaluation of the water potential of solutions of polyethylene glicol 8000 both in the absence and presence of other solutes. Plant Physiol., v. 72, n. 1, p. 66-70, 1983.

NANDULA, V. K. et al. Factors affecting germination of horseweed (Conyza canadensis). Weed Sci., v. 54, n. 5, p. 898-902, 2006.

NISENSOHN, L. et al. Malezas de reciente difusión en los agroecosistemas de la región sojera núcleo. Rosario: Facultad de Ciencias Agrarias. Universidad Nacional de Rosario, 2007. 104 p.

OLIVEIRA, M. J.; NORSWORTHY, J. K. Pitted norningglory (Ipomoea lacunosa) germination and emergence as affected by environmental factors and seeding depth. Weed Sci., v. 54, n. 5, p. 910-916, 2006.

REDDY, K. N.; SINGH, M. Germination and emergence of Hairy beggarticks (Bidens pilosa). Weed Sci., v. 40, n. 2, p. 195-199, 1992.

RODRIGUEZ, N. E.; RAINERO, H. P. Malezas nuevas? o viejas que se adaptan a los nuevos sistemas. Estación Experimental Agricola Manfredi- INTA. Córdoba. Argentina. 2004. 12 p. (Boletin, 1)

SADEGHLOO, A. et al. Seed germination and seedling emergence of velvetleaf (Abutilon theophrasti) and barnyardgrass (Echinochloa crus-galli). Planta Daninha, v. 31, n. 2, p. 259-266, 2013.

SMITH, C. A. et al. Arrowleaf sida (Sida rhombifolia) and prickly sida (Sida spinosa): germination and emergence. Weed Res., v. 32, n. 2, p. 103-109, 1992.

SOBRERO, M. T.; CHAILA, S. Manual digital de malezas del noroeste argentino. Santiago del Estero: 2006. CD ROM

SOLANO, F. et al. Germination, growth and development of spurred anoda. Weed Sci., v. 22, n. 4, p. 353-354, 1976.

SOUZA FILHO, A. P. S. et al. Seed germination in weeds from cultivated pasture areas: Mimosa pudica and Ipomoea asarifolia. Planta Daninha, v. 19, n. 1, p. 23-31, 2001.

ZHANG, Y. et al. Germination characteristics of Artemisia ordosica (Asteraceae) in relation to ecological restoratio in northern China. Canad. J. Bot., v. 83, n. 8, p. 1021-1028, 2005. 